\title{
Macroporous Silicagel Substrate for Stereoregular Template Polymerization of Methacrylic Acid Using Stereocomplex Assembled Thin Films
}

\author{
By Hiroharu AJIRO, ${ }^{1,2}$ Daisuke KAMEI, ${ }^{1}$ and Mitsuru AKASHI ${ }^{1,2, *}$
}

KEY WORDS: Template Polymerization / Stereospecific Polymerization / Poly(methacrylic acid) / Macroporous Silicagel /

Controlling polymer structure is important, because the chemical and physical characteristics of a polymer depend on its molecular weight distribution and stereoregularity. Polymerpolymer interactions have received much attention due to the possible application as a precise reaction control. For example, isospecific and syndiospecific template polymerizations of methyl methacrylate (MMA) have been reported with the use of stereocomplex formation in the presence of one stereoregular polymer. ${ }^{1,2}$ However, van der Waals interactions between isotactic- (it-) and syndiotactic- (st-) poly(methyl methacrylate)s (PMMA) exert an unfavorable effect against the formation of a stereoregular polymer during radical polymerization in solution, because the polymer chain moves constantly with thermal motion. Recently, molecularly porous thin films were employed for stereoregular template polymerization, which were prepared by the alternative layer-by-layer assembly of $i t$-PMMA/st-poly(methacrylic acid) (PMAA) and the subsequent extraction of one polymer from the stereocomplex film. ${ }^{3,4}$ It was a breakthrough for the precise control of radical polymerization on MMA and methacrylic acid (MA). This new stereocontrol approach is significant, because conventional anionic polymerization requires restricted polymerization conditions and organometal reagents. Porous itPMMA thin film formation, st-PMAA recognition, and stereoregular template polymerization of MA were investigated preliminarily on a quartz crystalline microbalance (QCM) substrate at the nano gram order. In order to manufacture stereoregular PMMAs with the aforementioned template polymerization method, a more efficient reaction field is crucial. Although silica gel (diameter: $1.6 \mu \mathrm{m}$ ) was used as a substrate because of its larger surface area, ${ }^{3}$ the issues still remain. For example, the aggregation of the silicagels bearing thin polymer films prevents good dispersion, which is required for an efficient reaction field. The smaller the silicagel is, the greater the reaction efficiency, but this makes it difficult to recover the silicagels completely by centrifugation after several dozen alternative immersion steps.

Therefore, we selected a macroporous silicagel (diameter: $7 \mu \mathrm{m}$, pore: $100 \mathrm{~nm}$ ) as a suitable substrate for template polymerization in this study. Macroporous silicagel is widely used, for example as the supporting for material of a stationary phase in high-performance liquid chromatography (HPLC). ${ }^{5}$ Packed column is possible to be applied for repeatedly available polymerization container. Herein, we report the template polymerization of MA on a porous it-PMMA thin film formed on macroporous silicagels, and discuss the curvature effect and reproducibility.

\section{EXPERIMENTAL}

\section{Materials}

Macroporous silicagel (diameter: $7 \mu \mathrm{m}$, pore: $100 \mathrm{~nm}$ ) was purchased from Daiso, Co., LTD. MMA (Tokyo Chemical Industry Co., LTD.), MA (Tokyo Chemical Industry Co., LTD.), trimethylsilylmethacrylate (Aldrich), toluene (Tokyo Chemical Industry Co., LTD.) were distilled with calcium hydride. 2,2'-Azobis(2-methylpropionamidine) dihydrochloride (V-50) (Wako) was used without further purification. itPMMA for Layer-by-layer assembly in this study was synthesized by anionic polymerization of MMA in toluene at $-78^{\circ} \mathrm{C}$ for $5 \mathrm{~d}$ with $t$ - $\mathrm{BuMgBr}^{6}$ (mm: $m r: r r=96: 3: 1, M_{\mathrm{n}}=$ 36,000 , PDI $=1.20$ ). st-PMAA for Layer-by-layer assembly was synthesized by anionic polymerization of trimethylsilylmethacrylate with $t$-BuLi/bis(2,6-di-tert-butylphenoxy)methyl aluminum, ${ }^{7}$ and the obtained polymer was methylated by diazomethane to be characterized (mm:mr:rr $=1: 5: 94, M_{\mathrm{n}}=$ 33,700 , PDI $=1.45$ ). Tacticities of polymers were determined by ${ }^{1} \mathrm{H}$ NMR spectra of alpha methyl protons in nitrobenzene- $d_{5}$ at $110^{\circ} \mathrm{C}$. Molecular weights and polydispersities were determined by SEC in THF at $40^{\circ} \mathrm{C}$ with PMMA standard.

\section{Preparation of Porous it-PMMA Thin Film}

Stereocomplex was prepared using it-PMMA $\left(M_{\mathrm{n}}=\right.$ $36,000)$ and st-PMAA $\left(M_{\mathrm{n}}=32,400\right)$, which were obtained by anionic polymerizations. The porous it-PMMA thin film on macroporous silicagel was typically prepared as follow. In plastic tube with cap, a $2 \mathrm{~g}$ of macroporous silicagel was introduced. $40 \mathrm{~mL}$ of it-PMMA in acetonitrile with

\footnotetext{
${ }^{1}$ Department of Applied Chemistry, Graduate School of Engineering, Osaka University, 2-1 Yamada-oka, Suita 565-0871, Japan

${ }^{2}$ The Center for Advanced Medical Engineering and Informatics, Osaka University, 2-2 Yamada-oka, Suita 565-0871, Japan

*To whom correspondence should be addressed (Tel: +81-6-6879-7356, Fax: +81-6-6879-7359, E-mail: akashi@chem.eng.osaka-u.ac.jp).
} 
Table I. Template polymerization of methacrylic acid

\begin{tabular}{ccccccc}
\hline Entry & $\begin{array}{c}\text { Solvent } \\
\text { for st-PMAA } \\
\text { MeCN:water }\end{array}$ & $\begin{array}{c}\text { Reuse } \\
\text { cycle }\end{array}$ & $\begin{array}{c}\text { Yield }^{\mathrm{mg}}(\%) \\
\mathrm{mg}^{2}\end{array}$ & $\begin{array}{c}\text { Tacticity } \\
m m: m r: r r\end{array}$ & $\begin{array}{c}M_{\mathrm{n}}{ }^{\mathrm{e}} \\
\times 10^{3}\end{array}$ & PDI $^{\mathrm{e}}$ \\
\hline 1 & $40: 60$ & - & $44(5 \%)$ & $1: 10: 89$ & 29.5 & 2.84 \\
$2^{\mathrm{b}}$ & $40: 60$ & Second & $28(3 \%)$ & $1: 11: 88$ & 28.9 & 2.53 \\
$3^{\mathrm{b}}$ & $40: 60$ & Third & $36(4 \%)$ & $1: 11: 88$ & 30.7 & 2.72 \\
$4^{\mathrm{b}}$ & $40: 60$ & Fourth & $28(3 \%)$ & $1: 8: 91$ & 31.2 & 2.66 \\
5 & $20: 80$ & - & $36(4 \%)$ & $3: 8: 89$ & 28.4 & 2.30 \\
\hline
\end{tabular}

a Methacrylic acid $=0.9 \mathrm{~mL}$. Initiator $=0.143 \mathrm{~g}$. Solvent $=$ water,

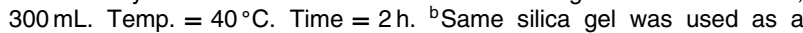
recycle three times. ${ }^{\mathrm{c}}$ Recovered as a $\mathrm{NaOH}$ aq.-soluble part. ${ }^{\mathrm{d}}$ Determined by ${ }^{1} \mathrm{H}$ NMR of alpha-methyl group of PMMA, which was derived from the

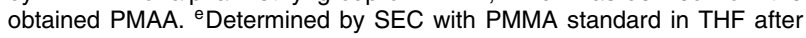
methylation.

0.017 unitM was then introduced into the tube to rotate mildly for $15 \mathrm{~min}$. After centrifugation, supernatant polymer solution was separated from macroporous silicagel, and washed with acetonitrile twice. Similarly, st-PMAA in acetonitrile/water $(4 / 6, \mathrm{v} / \mathrm{v})$ with 0.017 unitM was treated to form stereocomplexes of st-PMAA/it-PMMA on macroporous silicagel substrate. Those alternative immersions were repeated twenty cycles (Table I, entry 1-4). The same procedure was achieved to prepare the other stereocomplex on macroporous silicagel, except for st-PMAA in acetonitrile/water $(2 / 8, \mathrm{v} / \mathrm{v})$ and washing water for st-PMAA steps (Table I, entry 5). Then, $0.01 \mathrm{M} \mathrm{NaOHaq}$. was used to extract st-PMAA from the both stereocomplex, which results in the it-PMMA ultrathin film with stereoregular (st-PMAA) nanospace. The extraction was confirmed by the IR spectral change of carboxyl group (supporting information Figure S1).

\section{Template Polymerization}

$0.9 \mathrm{~mL}$ of MA was dissolved in ultrapure water, and the porous it-PMMA thin film on macroporous silicagel were introduced. Dry nitrogen gas was handled for more than $15 \mathrm{~min}$ before use, and then V-50 was added under nitrogen atmosphere to heat at $40{ }^{\circ} \mathrm{C}$ for $2 \mathrm{~h}$. The mixture was cool down to stop reaction. Macroporous silicagel was washed by water to recover PMAA, which was produced in solution. Then PMAA which was synthesized in porous it-PMMA thin film was extracted by $0.01 \mathrm{M} \mathrm{NaOHaq}$. The macroporous silicagel with porous thin film was used for three times.

\section{RESULTS AND DISCUSSION}

Figure 1 shows a schematic illustration of stereocomplex (itPMMA/st-PMAA) formation, porous it-PMMA film preparation, and the subsequent template polymerization of MA, together with SEM images of the silicagel. After 10 cycles of alternative immersion between the it-PMMA and st-PMAA solution, the it-PMMA/st-PMAA stereocomplex would be present on the surface. It is not surprising that holes in the silicagel could still be clearly observed, because the width of it-PMMA/st-PMMA stereocomplex is around $2 \mathrm{~nm}$ as reported elsewhere. ${ }^{8}$ These holes were also recognized even after 20
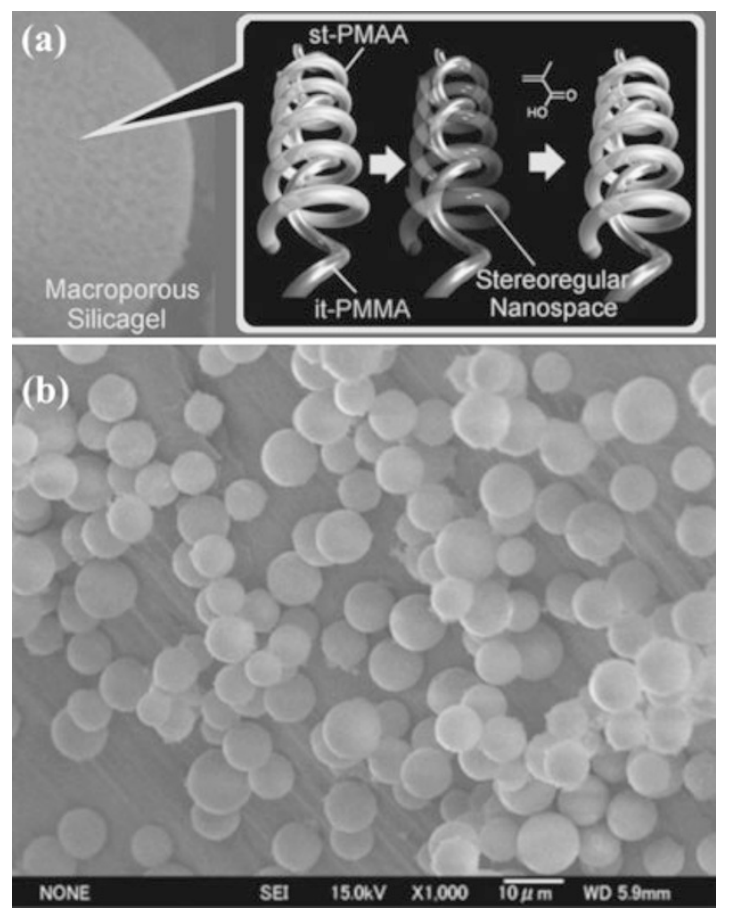

Figure 1. Schematic illustration of stereoregular template preparation for stPMAA (a). and a SEM image of the macroporous silicagel with itPMMA/st-PMAA stereocomplex film (b). White bar indicate $10 \mu \mathrm{m}$.

cycles of alternative immersion, although very small amount of polymer which are not adsorbed onto the silicagel was also observed (supporting information, Figure S2). Due to the washing processes of silicagel against the existing it-PMMA or st-PMAA, these polymers were assumed to be stereocomplexes. During the st-PMAA template (porous it-PMMA film) preparation with the subsequent extraction of st-PMAA, the holes in the silicagel were not completely covered with the polymers, but the silicagel surface became smoother and the hole size tended to be smaller; these findings were confirmed by SEM. This suggests that the polymer solution could permeate inside each silicagel particle, and a template field could also be created inside the macroporous silicagel.

The syndiospecific template polymerization of MA with the it-PMMA film on the silicagel was successfully achieved (Table I). ${ }^{3}$ The template polymerization with twenty cycles of alternative assembly on $2 \mathrm{~g}$ of macroporous silicagel resulted in $44 \mathrm{mg}$ (5\% yield) of PMAA, whereas $740 \mathrm{mg}$ of PMAA (81\% yield) was recovered from the solution. Previously, it has been reported that about $1.4 \times 10^{-6} \mathrm{mg}(1600 \mathrm{~Hz})$ of it-PMMA/ st-PMAA stereocomplex was adsorbed onto a QCM $(9 \mathrm{~mm}$ diameter) at 5 cycles of assembly. ${ }^{9}$ Assuming that the same amount of stereocomplex was adsorbed onto the macroporous silicagel, $2.6 \mathrm{~g}$ was estimated as the amount of stereocomplex film in this study, because a $60 \mathrm{~m}^{2}$ surface area of macroporous silicagel $\left(30 \mathrm{~m}^{2} / \mathrm{g}, 2 \mathrm{~g}\right)$ was used for 20 cycles of assembly. Taking the $1 / 2$ ratio of $i t$-PMMA/st-PMAA into account, the efficiency of the template polymerization was calculated to $3 \%$. However, the amount adsorbed onto the silicagel should be much smaller than the theoretical value, because the surface 


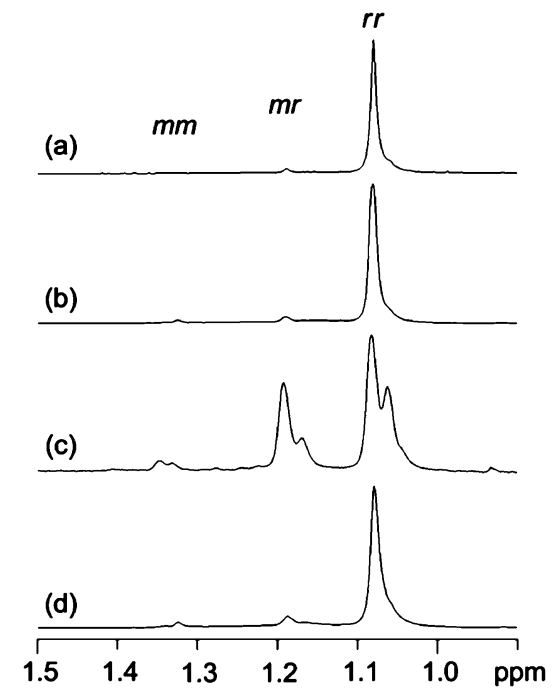

Figure 2. ${ }^{1} \mathrm{H}$ NMR spectra of synthesized poly(methacrylic acid) at $110^{\circ} \mathrm{C}$ in nitrobenzene- $d$ after methylation with diazomethane. st-PMMA obtained by anionic polymerization (a), PMMA extracted from stereocomplex thin film on macroporous silicagel at preparation of template reaction field (b), PMMA collected from solution after polymerization (c), and PMMA synthesized with porous it-PMMA on silicagel $(d)$.

energy of glass on macroporous silicagel is much smaller than that of gold on a QCM. Furthermore, the molecular weight $\left(M_{\mathrm{n}}=26,000\right)$ during the extraction procedure was smaller than that of st-PMAA used during stereocomplex formation (supporting information, Figure S3), and the molecular weight of synthesized PMAA was similar to that of the extracted PMAA. Taken together, these findings imply that not all stereocomplex film were effective for template field creation deep inside the pores, however, the template polymerization proceeded along the nano space.

A lack of syndiotacticity was observed in PMMA recovered from supernatant in the previously reported template polymerization, ${ }^{3}$ which was considered to be a result of the excess adsorption of $r r$-triads to the template thin film. However, the tacticity of the PMAA collected from solution in this study was atactic after treatment with diazomethane (Figure 2c), which is a similar value as the typical PMAAs obtained with radical methods. This is likely the result of the large amount of monomer polymerized as compared to the template thin film. On the other hand, PMAA synthesized on the macroporous silicagel was confirmed as highly syndiotactic (Figure 2d), suggesting that the stereoregularity of the PMAA was influenced by the it-PMMA film on the macroporous silicagel. The molecular weight of PMAA synthesized on the macroporous silicagel $\left(M_{\mathrm{n}}=28,000\right)$ was similar to the initially extracted st-PMMA $\left(M_{\mathrm{n}}=26,000\right)$, and smaller than that collected from solution $\left(M_{\mathrm{n}}=46,000\right)$, which implies that the polymerization circumstances were quite different.

$\mathrm{Next}$, the macroporous silicagel was maintained at $-20^{\circ} \mathrm{C}$ for $2 \mathrm{~d}$, and then the template polymerization was repeated two times (Table I, entries 2 and 3, Figure 3a and 3b). Another $13 \mathrm{~d}$ late, the template polymerization was performed again

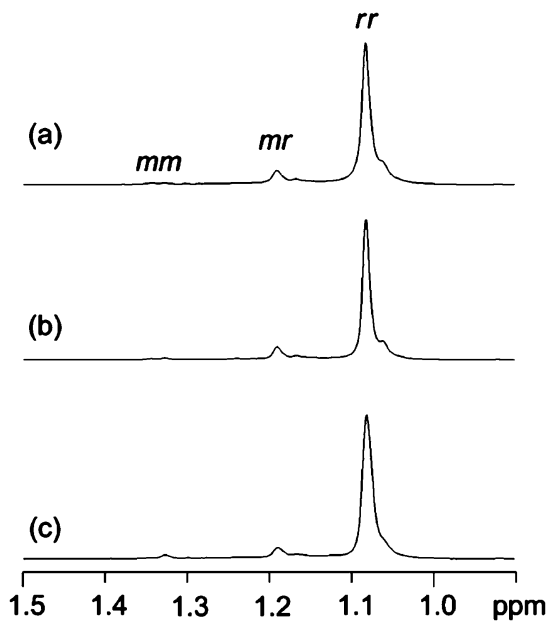

Figure 3. ${ }^{1} \mathrm{H}$ NMR spectra of synthesized poly(methacrylic acid) on the reused it-PMMA film after methylation by diazomethane (in nitrobenzene- $d, 110^{\circ} \mathrm{C}$ ). Secondly synthesized PMAA (a), thirdly synthesized PMAA (b), and fourthly synthesized PMAA (c).

(Table I, entry 4, Figure 3c). These results demonstrate that the polymerization field was stable for recycled use, because the highly syndiotactic selective polymerization was repeatedly observed.

Finally, the alternative assembly of it-PMMA/st-PMAA onto macroporous silicagel with 40 cycles was examined with acetonitrile/water $(2 / 8, \mathrm{v} / \mathrm{v})$ for the $s t$-PMAA solvent (Table I, entry 5). The reused template polymerization on the macroporous silicagel provided almost the same yield. Solvent effect on the amount of stereocomplex formation by layer-by-layer was reported. ${ }^{10}$ When acetonitrile/water (3/7, v/v) was employed, the stereocomplex amount decrease almost twothirds of that with acetonitrile/water (4/6). Thus, the result (Table I, entry 5) is understandable because of the solvent effect and twice layer-by-layer assembly. The molecular weights were controlled, and they were highly syndiotactic ( $r r \sim 90 \%$ ) as measured by ${ }^{1} \mathrm{H}$ NMR after methylation with diazomethane (supporting information, Figure S4) for all of the obtained PMAAs (Table I).

These reproducible results suggest that the template polymerization of methacrylic acid with it-PMMA/macroporous silicagels has practical applications for manufacturing using HPLC systems.

Electronic Supporting Information Available: Figures S1, S2, $\mathrm{S} 3$, and S4. These materials are available via. the Internet at http://www.spsj.or.jp/c5/pj/pj.html.

Received: August 22, 2008 Accepted: September 20, 2008 Published: November 6, 2008

\section{REFERENCES}

1. R. Buter, Y. Y. Tan, and G. Challa, J. Polym. Sci.: Part A-1, 10, 1031 (1972).

2. R. Buter, Y. Y. Tan, and G. Challa, J. Polym. Sci., Polym. Chem. Ed., 
11, 1003 (1973).

3. T. Serizawa, K. Hamada, and M. Akashi, Nature, 429, 52 (2004).

4. T. Serizawa and M. Akashi, Polym. J., 38, 311 (2006).

5. Y. Okamoto and E. Yashima, Angew. Chem., Int. Ed., 37, 1020 (1998).

6. K. Hatada, K. Ute, K. Tanaka, T. Kitayama, and Y. Okamoto, Polym. J., 17, 977 (1985).

7. T. Kitayama, S. He, Y. Hironaka, T. Iijima, and K. Hatada, Polym. J.,
27, 314 (1995).

8. J. Kumaki, T. Kawauchi, K. Okoshi, H. Kusanagi, and E. Yamshima, Angew. Chem., Int. Ed., 46, 5348 (2007).

9. T. Serizawa, K. Hamada, T. Kitayama, and M. Akashi, Angew. Chem., Int. Ed., 42, 1118 (2003).

10. T. Serizawa, K. Hamada, T. Kitayama, K. Katsukawa, K. Hatada, and M. Akashi, Langmuir, 16, 7112 (2000). 